\title{
Maternidad universitaria y el impacto generado al asumir esta doble condición
}

\author{
Cristina Criollo- Espín'; Silvia Altamirano -Altamirano²
}

Fecha de recepción: 25 de Julio 2018

Fecha de aceptación: 7 de Septiembre 2018

\section{Resumen}

Este artículo pretende identificar el impacto que genera el asumir esta doble condición de madre estudiante. Un estudio descriptivo, cuya muestra fue de 50 estudiantes madres en la facultad de Jurisprudencia y Ciencias Sociales de la Universidad Técnica de Ambato. Los resultados encontrados revelan un índice alto de embarazos no planificados (80\%), la primera fuente de apoyo sobre todo en mujeres solteras es la familia de origen, la conciliación de estos roles acarrea consecuencias respecto al desempeño académico en la estudiante madre, no hubo acompañamiento y apoyo de la pareja en las etapas de embarazo y maternidad referidas en mujeres solteras. El impacto fuerte que se genera debido a esta doble condición, es sobre todo en el estilo de vida de las universitarias, muchas de ellas no se encontraban preparadas para asumir estos roles lo que generó una ruptura en su círculo familiar y social.

Palabras clave: Maternidad, maternidad universitaria, impacto, doble condición, apoyo.

\section{Abstract}

This article aims to identify the difficulties and the impact that this double condition of student-mother produces. A descriptive study whose sample was 50 mother students from the Faculty of Jurisprudence and Social Sciences. The results found reveal: a high rate of unplanned pregnancies (80\%), the first source of support especially in single women is the family of origin. The conciliation of these roles has consequences for the academic performance of the mother student, there was not a right couple support in all the stages of pregnancy and maternity related to single women. The strong impact that is generated due to this double condition, is mainly in the life style of the university students, many of them were not prepared to assume these roles which generates a break in their family and social circle

Keywords: Maternity, university maternity, impact, double condition, support.

\section{Introducción}

La influencia de una cultura patriarcal que impera desde tiempos sociohistóricos hasta la actualidad cuyo discurso ha sido alimentado de distintas fuentes como la religión, la medicina y la filosofía (Soriano, 2012, p.339). Confiere en la feminidad y en el significado de la maternidad características que definen a la mujer como un ser inferior, débil, dependiente, sumiso, ser para otros, asexual complementada por un hombre superior y proveedor (Castillo, 2015, p.106; Cárdenas, Lay, Gonzales, Calderón y Alegría, 2010; Cristiani, Bertolotto, Anderson, Polinelli, Collavini, Seijo, y Delfino, 2015, p.13; Facio, 2002; Ferrer y Bosch, 2013; p.106; Lagarde, 1990, p.3).

Por ello, la corporalidad femenina responde a una imagen de maternidad: servicial, pasiva, emocional, que ama sin fronteras, ni límites, asociada al orden biológico, natural, y reproductivo propio de la mujer (Arvelo, 2004, p.93; Facio, 2002, p.65; Lagarde, 1990, p.6; Palomar, 2004, p.16; Palomar, 2005, p.36; Puyana y Mosquera, 2005, p.7) y contribuye al mito de mujer igual madre, cuyo significado ubica la permanencia de la mujer al ámbito privado (el hogar) y doméstico en consecuencia de ello, se espera que la mujer sea exitosa y realizada en dicho espacio (Cristiani, et, al, 2015:13; Facio, 2002, p.55; Ferrer y Bosch, 2013, p.107; Gonzáles, 2010, p.48; Lagarde, 1990, p.7; Lozano, Iglesias y Alonso, 2016, p.4; Rodríguez y Mancinas, 2016, p.18; Puyana y Mosquera, 2005, p.7; Sánchez, 2016, p.256,264).

Lo mencionado, es transmitido de generación a generación por estereotipos que transformados en axiomas legitiman el ejercicio de la mater- nidad, mediante procesos de socialización en los cuales se interiorizan formas de comportamiento, valores, normas y expectativas, reforzados a diario por agentes socializadores como son la familia, la comunidad, los amigos y medios de comunicación (Ferrer y Bosh, 2013, p.106; Rodríguez y Mancinas, 2016, p.18). Esta representación social y modelo de maternidad continua estable en la identidad femenina-en mayor o menor grado- en la sociedad ecuatoriana.

Es a partir del siglo $X X$, que diferentes momentos sociohistóricos contribuyen a la salida de la mujer al espacio público, que tradicionalmente era exclusivo para el hombre, empezando con la conquista de sus derechos civiles (Pizani, 2009; p.455), la incorporación masiva a la educación y al campo laboral, con ello el acceso y uso de métodos anticonceptivos para la planificación familiar. Si bien es cierto, datos según el INEC (Instituto Nacional de Estadística y Censos del Ecuador) refieren que nueve de cada diez mujeres, entre 15 y 49 años conoce de métodos anticonceptivos $(90,35 \%)$ pero apenas cuatro de cada 10 utiliza alguno $(49,8 \%)$ (Ferreira, et al, 2010, p.60).

Estos momentos, generan en parte una redefinición de roles femeninos, pero aun manteniéndose en parte el rol tradicional propio de la cultura patriarcal. A su vez aparecen nuevos proyectos de vida enfocados al desarrollo profesional, debido a ello, hoy en día la mujer busca generar un equilibrio entre su vida social, laboral, educativa, crianza de sus hijos/as y actividades domésticas, involucrando en ello a otros agentes sociales que contribuyan a tal equilibrio en ambos espacios público y privado (Cristiani, et, al, 2015, p.13-14; Peña, 2015, p.2).

Razón por la cual se empiezan a considerar nuevas tipologías de familia (Moroto, García y Rodríguez, 2004, p.14), muy aparte de la familia tradicional nuclear. Aparecen en los hogares la jefatura femenina asumida por madres solteras, divorciadas o separadas y hay un incremento de las familias de tipología monoparental (Cristiani, et, al, 2015, p.13; Miller y Arvizu, 2016, p.29).

Por otro lado, es importante hacer referencia a la división sexual del trabajo, es muy notable el alcance de la mujer al espacio público al formar parte del campo laboral (8 horas diarias) o cursar estudios académicos. Pero el trabajo que realiza dentro del hogar, continúa siendo invisibilizado en la sociedad. Datos estadísticos demuestran que en el Ecuador de unas 60,8 horas en promedio del trabajo semanal que realiza la mujer, el 40,2 pertenece al trabajo no remunerado o invisible, mientras que el 20,6 equivale al trabajo remunerado (CEPAL, 2016). Dando a notar que la mujer dedica mayor número de horas al trabajo doméstico dentro del hogar contrarias al tiempo que dedica para el trabajo remunerado.

Como consecuencia de lo referido, se genera en la mujer una doble carga o doble jornada como lo menciona la literatura científica (Castañeda, 2015; Guzmán, 2017, p.81; Lagarde, 1990, p.7; Mora, 2013; Moroto, García y Rodríguez, 2014, p.14; Palomar, 2004, p.14, Peña, 2015, p.4). Debido a los estereotipos culturales que exigen a la mujer el desempeño de las actividades del hogar y sobre esto, tareas concernientes al cuidado de los hijos/as. (Peña, 2015, p.4).

Este es el caso, que es vivenciado y experimentado por las mujeres madres que cursan estudios universitarios en el Ecuador, quienes buscan a partir de la educación mejores oportunidades sociales y laborales, tanto para ellas como para sus hijos (Guzmán, 2017, p.81).

\footnotetext{
Trabajadora Social. Egresada de la Universidad Técnica de Ambato. Facultad de Jurisprudencia y Ciencias Sociales. Ambato- Ecuador. Email: criscriollo93@gmail.com

'Mg en Trabajo Social Familiar. Mg en Trabajo Social en educación y especialista en Gestión Social. Docente tutor de la Universidad Técnica de Ambato. Facultad de Jurisprudencia y Ciencias Sociales. Carrera de Trabajo Social. Ambato- Ecuador. Email: sj.altamirano@uta.edu.ec
} 
Dentro del contexto universitario, el embarazo en estudiantes es considerado una situación devenida o no esperada, porque en su mayoría son embarazos no planificados (Estupiñan y Rodríguez, 2009, p.995; Guzmán, 2017:81) y generan un impacto profundo en las relaciones de familia, pareja y amigos, debido a que muchas de ellas no se encuentran preparadas psicológicamente y en la mayoría de los casos económicamente para ejercer la maternidad (Cimino, Duran, Herbage, Palma y Roa, 2014, p.24; Estupiñán y Vela, 2012, p.538; Domínguez, Peralta y Rincón, 2015, p.168). Los hijos/as aparecen en esta trayectoria como un hito que marcan momentos de fuertes dificultades y generan nuevas necesidades en la madre universitaria (Palomar, 2009, p.70).

Varios autores en sus investigaciones reportan que la maternidad tiende a afectar de diferente forma el proceso de aprendizaje y el desempeño académico, debido a que el embarazo, puerperio y lactancia son situaciones llevadas solamente por la mujer, lo que también se considera a su vez como una limitación más, para desempeñar sus estudios con normalidad (Estupiñán y Vela, 2012, p.538; Lozano, Iglesias y Alonso, 2016, p.3). Esto también genera a su vez importantes rupturas en su círculo social y de ocio, provocado por la demanda de tiempo que compete el ejercicio de la maternidad y las responsabilidades académicas e incluso hay un riesgo alto de abandonar la carrera universitaria, en especial cuando se está iniciando los primeros semestres de estudio (Cimino, et al, 2014, p.24; Estupiñán y Rodríguez, 2009, p.990, 993; Estupiñán y Vela, 2012, p.538; Palomar, 2009, p.71).

Esta doble condición genera otras consecuencias que afectan la calidad de vida de la madre universitaria como el abandono de oportunidades y renuncia de sueños académicos, la suspensión de congresos, seminarios conferencias dentro y fuera de la ciudad, aplazo de materias o semestres (Palomar, 2009, p.71), conlleva a su vez una reorganización de hábitos académicos y sociales (Cimino, et al, 2015, p.36), se produce en ellas un agotamiento físico y psicológico, soledad y baja autoestima (Estupiñán y Vela, 2012, p.542, 546).

Lo que implica buscar formas para conciliar estos roles de madre-estudiante, y con ello generar un equilibrio que contribuya a minimizar la carga y peso que con lleva los estudios universitarios con el ejercicio de la maternidad, ya que por una parte se encuentra su deseo personal de estudiar para adquirir una profesión a futuro que contribuya a una mejor calidad de vida y por el otro debe cumplir el rol de madre de acuerdo con las expectativas personales y sociales ligadas a la maternidad (Domínguez, Martínez, Carrera y De la Fuente, 2015, p.383; Estupiñán y Rodríguez, 2009, p.990; García, Kral, Acuña y Preciado, 2007, p.3; Guzmán, 2017, p.82; Lozano, Iglesias y Alonso, 2016, p.3).

Para el logro de la conciliación de estos roles la madre universitaria genera una serie de estrategias, busca y demanda apoyo a terceros para el cuidado de sus hijos/as (Estupiñán y Rodríguez, 2009, p.993; Estupiñán y Vela, 2012, p.542; González, 2010, p.50) y de esta manera dar continuidad a sus estudios y evitar el abandono de la carrera.

Investigaciones refieren que la primera fuente de apoyo en la mayoría de los casos fue la madre de la estudiante, seguido de su familia de origen, quienes responden a tal demanda y necesidad de la estudiante y brindan apoyo de tipo económico generalmente para lidiar con los gastos que se requiere en la universidad y en la maternidad; y apoyo de tipo emocional con su comprensión y afecto, produciendo una sensación de bienestar que contribuye a superar las dificultades, reduce los niveles de ansiedad, estrés y depresión que se le presente y hacen que esta doble condición sea más llevadera (Cimino, et al, 2014, p.26; Domínguez, et al, p.168; Estupiñán y Vela, 2012, p.542,544; Estupiñán y Rodríguez, 2009, p.994).

Por otra parte, la universidad como fuente de apoyo es percibida por las madres universitarias de manera negativa, pues sienten el rechazo y desconsideración de parte de sus docentes y compañeros de clase, lo que dificulta la conciliación y el quehacer académico (Estupiñán y Vela, 2012, p.541).

Varias investigaciones revelan que en su mayoría de madres universitarias hay un número alto de aquellas que son solteras reproduciendo el estereotipo de madresolterismo (Domínguez, et al, 2015, p.168; Estupiñán y Rodríguez, 2009, p.988; Guzmán, 2017, p.81), menciona a su vez que el apoyo y acompañamiento de la pareja en la etapa de embarazo y maternidad es solo durante los primeros meses del recién nacido seguido del abandono (Cimino, et al, 2014, p.25; Domínguez, et al, 2015, p.168; Palomar, 2009, p.990) por lo tanto afrontan solas esta realidad junto con el apoyo de su familia de origen.

El papel que juega el apoyo en la conciliación de los roles de las estudiantes madres, contribuye a que se minimice la carga emocional y académica que sostiene al conciliar estos roles (Cimino, et al, 2014, p.26) y es fundamental para el logro de las metas profesionales en la estudiante y sobre todo aporta a que la estudiante no abandone sus estudios (Estupiñán y Vela, 2012, p.541).

Por todo lo anteriormente señalado, esta investigación pretende responder a tres cuestiones, en primer lugar, identificar el impacto generado en las estudiantes al asumir esta doble condición dentro de la universidad y sus implicaciones en su círculo familiar y social, en segundo lugar, el conocer como manejan y distribuyen su tiempo para conciliar estos roles., y en tercer lugar identificar cuanto apoyo de la familia y de qué tipo las estudiantes reciben para tal conciliación y dentro de ello el apoyo y acompañamiento de la pareja en las etapas de embarazo y maternidad.

La evidencia empírica encontrada revela que este estudio no se ha realizado en la provincia de Tungurahua-Ecuador y se desconocen publicaciones de temas similares por autores ecuatorianos en revistas de prestigio.

\section{Metodología}

La presente investigación tiene un enfoque cuantitativo (Hernández, Fernández, y Baptista, 2014) y fue llevada a cabo con la aplicación de una encuesta cuyas preguntas identifican datos sociodemográficos, uso del tiempo y condición de la maternidad universitaria.

Este estudio se lo ha realizado en la Facultad de Jurisprudencia y Ciencias Sociales de la Universidad Técnica de Ambato-Ecuador, un muestreo por conveniencia para efectuar el presente estudio descriptivo orientado a identificar las dificultades que vivencian las madres universitarias y su impacto al asumir esta doble condición de estudiante-madre.

Para ello las estadísticas informan que en la facultad mencionada existe un total de 90 estudiantes matriculadas que cumplen con esta doble condición, de las cuales un $55,5 \%$ que equivale a 50 madres quienes fueron encuestadas.

\section{Resultados}

Los resultados obtenidos de la encuesta aplicada revelan que el $84 \%$ de estudiantes madres cursa Trabajo Social, 12\% Comunicación Social y un $4 \%$ pertenece a la carrera de Derecho, sus edades están comprendidas entre los 19 y 38 años, siendo la media de 23,9 años (SD 3,8a). El 26\% tiene 23 años, seguido del 16\% de encuestadas con 25 años.

El 32\% de estudiantes-madres se encuentran en décimo semestre, seguido de noveno semestre con el $20 \%$ es decir que a mayor semestre se da un incremento en la maternidad. El 12\% de madres universitarias corresponde a los semestres de sexto y segundo, el 10\% equivale a los semestres de segundo, quinto y octavo y el $2 \%$ a primer y tercer semestre.

\section{Datos sociodemográficos}

El $86 \%$ de madres universitarias tiene un hijo o hija, el $12 \%$ tiene dos hijos/as y el $2 \%$ tres hijos/as. De los cuales un $28 \%$ equivale a hijos menores de un año el 18\% equivale a hijos de dos años el 14\% tiene hijos de 5 años, el 12\% tiene hijos de edades de 7 años, el 10\% tiene hijos de las edades de 3 y 4 años, el $4 \%$ de 6 años y finalmente el $2 \%$ que refiere a las edades de 12 y 15 años de edad, generalmente este último dato corresponde a las madres universitarias cuya edad sobrepasa los 25 años.

El $36 \%$ de las madres universitarias viven actualmente con su familia nuclear de ese porcentaje solo el $4 \%$ conjuntamente con su pareja; el $14 \%$ conforman las familias de tipo monoparental, siendo la madre de la estudiante la cabeza del hogar en el $49 \%$ de este tipo de hogares. El $32 \%$ han conformado sus propios hogares con estructura nuclear, otro $6 \%$ con una estructura extensa. Un $4 \%$ viven solas con sus hijos. 
En cuanto a situación laboral se encuentra un porcentaje alto del $62 \%$ mencionan que no tienen experiencia laboral. Seguido de un $24 \%$ igual se encuentran buscando trabajo. El $8 \%$ de estudiantes tienen un trabajo asegurado al IESS mientras que el $6 \%$ mantiene un trabajo ocasional. Entre quienes tienen trabajo estable, la experiencia acumulada es de 7-8 años $2 \%$ de madres universitarias.

En relación a su estado civil, el $44 \%$ son solteras, el $38 \%$ casadas y el $18 \%$ informa uniones de hecho. Entre las solteras el 18\% informa mantener una relación actual de la cual sus hijos/as el $12 \%$ no son fruto de esa relación. De las casadas el 30\% refiere que sus hijos/as si son fruto de su relación actual en este caso son sus esposos y de las mujeres que mantienen una unión de hecho sus hijos/as son fruto de esa relación.

\section{Datos del uso del tiempo.}

En cuanto a las horas semanales que dedica la estudiante madre a las tareas domésticas y cuidado de sus hijos/as el 58\% dedica de 10 a 50 horas semanales, el $26 \%$ dedica de 55 a 100 horas y finalmente un $16 \%$ menciona que dedica entre 110 hasta 170 horas a los semanales exclusivamente a las tareas domésticas y cuidado de los hijos.

Las actividades que realizan las madres universitarias en su tiempo libre son en mayor número las tareas del hogar y cuidado de los hijos $66 \%$ un $31 \%$ a pasar tiempo de ocio con la familia y un 3\% a actividades de refuerzo académico como el seguir un curso o asistir a un seminario.

\section{En relación a la condición de embarazo y maternidad:}

8 de cada 10 embarazos en estudiantes no fueron planificados $(80 \%$ no planificados y $20 \%$ de embarazos planificados), de los cuales el $62 \%$ de estudiantes tuvieron miedo de comunicar su embarazo, un 92\% tuvo miedo de comunicar su embarazo a sus padres.

En relación al uso de métodos anticonceptivos el 90\% de mujeres madres respondieron que no fueron obligadas a utilizar métodos anticonceptivos, mientras que el $10 \%$ respondieron que, si fueron obligadas a alguno, de las cuales el $4 \%$ fueron obligadas por sus madres, el otro $4 \%$ por sus parejas y el $2 \%$ obligadas por ambos padres. Todas ellas fueron obligadas a utilizar métodos de anticoncepción después de su primer embarazo.

Respecto al matrimonio por conveniencia de su embarazo, el 68\% refieren que no se casaron por causa de su embarazo, mientras que $30 \%$ refieren que si lo hicieron. De las cuales el $74 \%$ no fueron presionadas para contraer matrimonio mientras que el $20 \%$ menciona que, si hubo presión para casarse o unirse, de ellas el $12 \%$ fueron presionadas por sus padres, el $4 \%$ fue la familia de la pareja y el $2 \%$ fueron presionadas por los padres de la pareja, y por otras personas ajenas a la familia.

En cuanto al acompañamiento por parte de la pareja durante el embarazo y maternidad, el $84 \%$ refieren que su pareja brindó acompañamiento en el proceso de puerperio y maternidad mientras que el $16 \%$ refiere que no fue acompañada en ninguna etapa de su embarazo y parto. En relación al tiempo de acompañamiento de la pareja el 60\% menciona que fue desde el nacimiento del bebé hasta la actualidad, seguido del $22 \%$ quienes no recibieron ningún acompañamiento, el $8 \%$ fue solo los primeros tres meses de vida del bebe y el $2 \%$ de 4 a 8 meses del recién nacido y el otro $2 \%$ corresponde al acompañamiento solo durante los 9 meses hasta el año del bebé. Seguido del abandono de la pareja.

Respecto a la afectación del embarazo o la maternidad en el rendimiento académico, el 34\% refiere que tanto la maternidad como el embarazo afectó a veces, el 26\% rara vez, 24\% refiere que nunca, un 10\% refiere que siempre y un $6 \%$ menciona casi siempre se vio afectado su rendimiento académico por causa del embarazo y maternidad.

De ello el 60\% refiere que el embarazo y maternidad limitó el tiempo para realizar tareas, el 52\% afectó en su asistencia a clases.

Para mirar cuantas estudiantes madres reciben apoyo de parte de su familia de origen y de la pareja, se procedió a realizar un cruce de tablas entre el estado civil y apoyo recibido. $Y$ de esta manera identificar a quienes reciben mayor o menor apoyo y de que fuente.
Tabla 1. Cuál es su estado civil ${ }^{*}$ Brinda ayuda económica para que pueda dar continuidad a sus estudios y los gastos que demanda la maternidad pareja actual

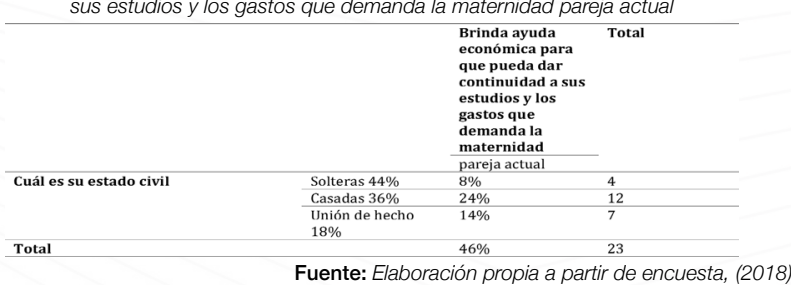

De las universitarias casadas (38\%) solo el 24\% informan recibir mayor apoyo económico por parte de su pareja para abastecer los gastos académicos y los de maternidad. Del $44 \%$ de solteras solo un $8 \%$ informa recibir apoyo de la pareja, y finalmente del $18 \%$ de madres que mantienen unión de hecho el 14\% informa recibir apoyo de la pareja.

Tabla 2. Cuál es su estado civi*Binda ayuda economica para que pueda dar continuidad

\begin{tabular}{|c|c|c|c|}
\hline & & $\begin{array}{l}\text { Brinda ayuda } \\
\text { económica para } \\
\text { que pueda dar } \\
\text { continuidad a sus } \\
\text { estudios y los } \\
\text { gastos que } \\
\text { demanda la } \\
\text { maternidad } \\
\text { Ambos padres }\end{array}$ & Total \\
\hline \multirow[t]{3}{*}{ Cuál es su estado civil } & Solteras $44 \%$ & $18 \%$ & 9 \\
\hline & Casadas 36\% & $16 \%$ & 8 \\
\hline & $\begin{array}{l}\text { Unión de hecho } \\
18 \%\end{array}$ & $10 \%$ & 5 \\
\hline Total & & $44 \%$ & 22 \\
\hline
\end{tabular}

Del $38 \%$ de universitarias casada, el 16\% informa que ambos padres brindan apoyo económico, el 16\% de mujeres unidas informan recibir ayuda de ambos padres y un $18 \%$ de solteras que es el porcentaje alto informan que ambos padres brindan ayuda de tipo económico.

\begin{tabular}{|c|c|c|c|}
\hline & & $\begin{array}{l}\text { Brinda ayuda } \\
\text { económica para } \\
\text { que pueda dar } \\
\text { continuidad a sus } \\
\text { estudios y los } \\
\text { gastos que } \\
\text { demanda la } \\
\text { maternidad } \\
\text { Madre }\end{array}$ & Total \\
\hline \multirow[t]{3}{*}{ Cuál es su estado civil } & Solteras $44 \%$ & $20 \%$ & 10 \\
\hline & Casadas $36 \%$ & $6 \%$ & 3 \\
\hline & $\begin{array}{l}\text { Unión de hecho } \\
18 \%\end{array}$ & $2 \%$ & 1 \\
\hline Total & & $28 \%$ & 14 \\
\hline
\end{tabular}

Las mujeres solteras son quienes mayor apoyo de la madre reciben con un $20 \%$ contrario a las mujeres casadas y unidas cuyo apoyo es bajo. Cabe recalcar en estos resultados encontrados y mediante la literatura analizada, la madre universitaria es quien demanda apoyo a sus redes como su pareja y su familia de allí el análisis de quien responde a esta demanda y entrega el apoyo solicitado.

En este estudio se encontró un número alto de universitarias que mencionan que sus embarazos no fueron planificados (80\%) coincide con la investigación de Guzmán, (2017, p.81) en México, basada en nuevas figuras estudiantiles, que reporta que las estudiantes universitarias no planificaron sus embarazos por ello se vieron enfrentadas a una nueva situación, para lo cual no estaban preparadas lo que dificultó su trayectoria universitaria, generando nuevas necesidades.

Esto a su vez da un valor significativo a los datos encontrados en el INEC, acerca del índice alto de embarazos no planificados, que nueve de cada diez mujeres de entre 15 y 49 años conoce métodos anticonceptivos $(90,35 \%)$ pero apenas cuatro de cada 10 lo utiliza $(49,8 \%)$ (Ferreira, et al, 2010:60). En relación al uso de método anticonceptivo el $90 \%$ de mujeres madres respondieron que no fueron obligadas a usarlo, mientras que el 10\% respondió que, todas ellas obligadas a utilizar métodos de anticoncepción después de su primer embarazo.

Otro punto encontrado referente a lo mencionado, es que se genera un incremento en la maternidad conforme avanzan los semestres, el $32 \%$ de madres estudiantes son de décimo semestre, seguido de noveno semestre con el 20\%, a diferencia de los semestres menores donde hay un porcentaje bajo de estudiantes madres. 
Referente a la doble jornada se encontró que la maternidad incide en el desempeño académico como manifiestan los porcentajes en los resultados de la encuesta, el 34\% cuya frecuencia fue "a veces" dirigido al rendimiento académico, el 60\% tuvo una limitación en su tiempo para realizar tareas, el $52 \%$ menciona faltas constantes a clases. Este hecho también es reportado en la literatura científica que refiere que la maternidad tiende a afectar de diferente forma el proceso de aprendizaje y el desempeño académico (Estupiñán y Vela, 2012, p.538; Lozano, Iglesias y Alonso, 2016, p.3).

Estos datos encontrados en mujeres estudiantes también podrían ser asociados con las estadísticas que manifiesta la CEPAL, 2016 acerca del trabajo remunerado y no remunerado con las mujeres que laboran, de las cuales las 60,8 horas en promedio del trabajo semanal que realiza la mujer, el 40,2 pertenece al trabajo no remunerado o invisible. Tal es el caso en mujeres madres universitarias, donde también se encontraron datos referentes al número de horas que dedican a las actividades del hogar y crianza de los hijos, el 58\% de estudiantes madres mencionaron que dedican entre 10 a 50 horas semanales, el $26 \%$ dedica de 55 a 100 horas y finalmente un $16 \%$ menciona que dedica entre 110 hasta 170 horas semanales, aquí cabe recalcar las horas que la estudiante permanece dentro de la universidad en una jornada de clases que son de 5 a 7 horas.

Lo expuesto, denota la doble condición de la universitaria y el impacto fuerte en el estilo de vida de las estudiantes, en su familia y de la pareja pues es notable la dificultad y consecuencias que se genera al momento de conciliar estos roles de madre- estudiante, el papel que juega el apoyo durante esta doble condición, contribuye a que se minimice la carga emocional y académica y genera un aporte a la mejor calidad de vida (Cimino, et al, 2014, p.26). Este apoyo es fundamental para el logro de las metas profesionales en la estudiante y sobre todo influye de manera positiva a que la estudiante no abandone sus estudios universitarios (Estupiñán y Vela, 2012, p.541).

\section{Conclusiones}

A modo de conclusión es evidente el impacto generado en la asunción de la doble condición de madre-estudiante de la muestra estudiada, y en los casos encontrados se suma el papel de esposa. Es una situación nueva en la vida de la universitaria para lo cual no se encuentra preparada, cuyas implicaciones producen una ruptura en su círculo familiar al ser un embarazo no planificado y social por la demanda de tiempo que conlleva la maternidad. Esto denota la perpetuidad y reproducción de los estereotipos de "buena madre", supeditando su formación profesional al rol superior de la maternidad asumida dentro del prototipo familiar vigente en la cultura patriarcal de la sierra ecuatoriana.

Las tareas del hogar y sobre todo el cuidado de los hijos/as en las estudiantes implican el mayor uso de horas semanales, esto limita el tiempo de dedicación al estudio, realización de tareas, asistencia a clases y en cierta parte es un factor más para el abandono de la carrera universitaria, sobre todo durante los primeros semestres. Este produce que la estudiante demande y busque apoyo a sus redes familiares y de esta forma lograr un equilibrio entre los estudios universitarios y la maternidad, por ello el papel que juega la familia y la pareja, influye de manera positiva en la vida de la estudiante, pues el apoyo recibido ya sea de carácter económico o en el cuidado de los hijos/as, contribuye al logro de metas profesionales y sobre todo que esta doble condición sea más llevadera reduciendo el estrés en la universitaria, la primera fuente de apoyo encontrada fue la madre de la estudiante, seguida de la familia de origen, en cuanto al acompañamiento y apoyo de la pareja durante el embarazo y crianza, fue mínimo seguido del abandono sobre todo en mujeres solteras contrario a mujeres casadas cuyo apoyo de la pareja es sobre todo de carácter económico para los gastos que compete entre estudios universitarios y maternidad.

Por otro lado, el altísimo índice de embarazos no planificados en estudiantes, pone el acento en dos asuntos de interés para el Trabajo Social en el ámbito universitario: el primero relacionado a la sexualidad aun vista como un tabú asociado a la planificación familiar, pese al conocimiento acerca de métodos anticonceptivos y al acceso a casas de salud y métodos de anticoncepción gratuitos que el Gobierno Ecuatoriano implementa para la sociedad; segundo relacionado a la permanencia del estereotipo de madresolterismo en mujeres jóvenes pues de igual forma hay un número alto de madres solteras en la Facultad. Y finalmente al dar a conocer la realidad que atraviesan las madres estudiantes a la sociedad, se pretende generar concientización tanto en las y los estudiantes como en las autoridades, enfatizando la intervención de la universidad como institución pública del Estado a la conciliación de estos roles con políticas sociales y proyectos donde estas realidades encontradas no sean obstáculo que interfiera en la trayectoria educativa y se evite de esta manera que las estudiante interrumpan sus estudios a causa de la maternidad o embarazo.

\section{Referencias}

Arvelo, L. (2004). Maternidad y paternidad, género y psicoanálisis. Otras Miradas, 4(2), 92-98.

Castillo, A. (2015). La práctica social de la maternidad y de la paternidad en jóvenes estudiantes de nivel superior: un acercamiento a las problemáticas cotidianas enfrentadas durante la vida académica. Revista Estudios sobre las Culturas Contemporáneas. 21(2), 103-123.

Cárdenas, M., Lay, S., Gonzales, C., Calderón, C., y Alegría, I. (2010). Inventario de Sexismo Ambivalente: Adaptación, validación y relación con variables psicosociales. Revista Salud y Sociedad. 1(2), 126-135.

Castañeda, M. (2015). Ser estudiantes, madres y padres una dualidad cotidiana. [Tesis de pregrado no publicada de antropología social]. Chile.

CEPAL. (2016). Trabajo no remunerado un aporte para la economía. Observatorio de igualdad de Género de América latina y el Caribe.

Cristiani, L., Bertolotto, M., Anderson, M., Polinelli, S., Collavini M., Seijo, F., y Delfino, M. (2015). Significado cultural de la crianza y de los roles materno y paterno. Aportes a la reflexión teórica. VI Congreso Internacional de Investigación y Práctica Profesional en Psicología XXI Jornadas de Investigación Décimo Encuentro de Investigadores en Psicología del MERCOSUR. Universidad de Quilmes. Argentina.

Cimino, J., Durán, B., Herbage, R., Palma, M., y Roa, J. (2014). Ser madre y estudiante universitaria en la Universidad de Santiago de Chile. Un estudio exploratorio acerca de las implicaciones psicosociales en el enfrentamiento de ambos roles. Revista de estudios cualitativos USACH (Universidad de Santiago de Chile), 1(1), 23-39.

Domínguez, A., Martínez, A., Herrera, H y Rincón, M. (2015). Impacto personal y escolar del embarazo y maternidad en estudiantes universitarios. Investigación y práctica en psicología del desarrollo, 1, 167-173.

Dominguez, A., Martínez, A., Carrera, P., y De la Fuente, C. (2015). Mirada en perspectiva de la maternidad no planeada en egresadas de psicología de una universidad pública. Investigación y práctica en psicología del desarrollo. 1, 383-389. México.

Estupiñán, M., y Rodríguez, L. (2009). Aspectos psicosociales en universitarias embarazadas. Revista Salud Pública, 11(6), 988-998.

Estupiñán, My Vela, D. (2012). Calidad de vida de madres adolescentes estudiantes universitarias. Revista Colombiana de Psiquiatría, 41(3), 536-549. 2 (2), 49-79.

Facio, A. (2002). Engenerando nuestras perspectivas. Otras Miradas,

Ferrer, V., y Bosch, E. (2013). Del Amor Romántico a La Violencia de Género. Para Una Coeducación Emocional En La Agenda Educativa. Revista de Curriculum y Formación de Profesorado. 17(1), 105-22.

Ferreira, C., García, K., Macías, L., Pérez, A., y Tomsich, C. (2010). Hombres y mujeres del Ecuador en Cifras III, serie de información estratégica. Ecuador: Ed. Ecuador.

García, M., Kral, K., Acuña, M., y Preciado F, (2007). La importancia del entorno social en la trayectoria escolar de estudiantes universitarios (gestantes, madres y padres). XXVI Congreso de la Asociación Latinoamericana de Sociología. Asociación Latinoamericana de Sociología, Guadalajara.

Gonzales, A. (2011). Reflexiones sobre el ejercicio actual de la maternidad. Revista Costarricense de Psicología. 29(43), 47-55.

Guzmán, C. (2017). Las nuevas figuras estudiantiles y los múltiples sentidos de estudios universitarios. Revista de la educación superior. 46(182), pp. 71-87. México. 
Hernández Sampieri, R., Fernández Collado, C., \& Baptista Lucio, P. (2014). Metodología de la investigación. (6a. Ed). México D.F.: Mc Graw - Hill.

Lagarde, M. (1990). Identidad Femenina, trabajo difundido por CIDHAL (Comunicación, intercambio y Desarollo Humano en América Latina, A.C.-México). México. Lozano, I., Iglesias, M., y Alonso, A. (2015). Conciliación estudiantil-familiar. Un estudio cualitativo sobre las limitaciones que afectan a las madres universitarias. Revista ARBOR Ciencia, Pensamiento y Cultura. 192(780), a341.

Miller, D., y Arvizu, V. (2016). Ser madre y estudiante. Una exploración de las universitarias con hijos y breves notas para su estudio. Revista de la educación superior. 1(177), 17-42.

Montes de Oca, V. (2000). Relaciones Familiares y redes sociales. En V. Montes de Oca (Ed.), Envejecimiento demográfico en México: retos y perspectivas (pp.289-326). México: Consejo Nacional de Población.

Mora, Esther. (2013). El paradigma género y mujeres en la historia del tiempo presente. Revista Historia Autónoma, (2). 143-160. Universidad de Murcia.

Moroto, G., García, M y Rodríguez, I. (2004). El reto de la maternidad en España: Dificultades sociales y sanitarias. Revista Gaceta Sanitaria. 18(5), 13-23. Barcelona.

Palomar, C. (2004). "Malas Madres": La construcción social de la maternidad, en Debate Feminista, 30, 12-34.

Palomar, C. (2005). Maternidad: Historia y Cultura. Revista La Ventana. (4), 35-67. México.

Palomar, C. (2009). Maternidad y mundo académico. Revista Alteridades. (19)38, 55-73. México.

Pizani, M. (2009). ¿Estado constitucional de derechos?: informe sobre derechos humanos. Los derechos humanos de las mujeres en el Ecuador. Quito-Ecuador. Ed. Abya-yala.

Puyana, Y., y Mosquera, C. (2005). Traer "hijos e hijas al mundo": significados culturales de la paternidad y la maternidad. Revista Latinoamericana de Ciencias Sociales, Niñez y Juventud, 3(2), 1-21 Colombia.

Peña, N. (2015). Introducir el género en las instituciones educativas: un desafío estratégico e impostergable. Boletín no. 51, 2-9. Colombia.

Rodríguez, L., y Mancinas, S. (2016). Nivel de sexismo y estereotipos de género en estudiantes de Trabajo Social Regiomontanas. Revista Internacional De Trabajo Social y Bienestar, (2), 17-30.

Sánchez, N. (2016). La experiencia de la maternidad en mujeres feministas. Revista Nómadas, (44), 255-267.

Soriano, M. (5,6 y 7 de marzo de 2012). La Transmisión de Ideas a lo Largo de la Historia: El discurso misógino del sistema patriarcal, En libro de actas del I Congreso Internacional de Comunicación y Género Sevilla. Dir. Juan Carlos Suarez Villegas; Comité organizador Irene Liberia Vayá y Belén Zurbano Berenguer (339-360). Sevilla: facultad de comunicación. Universidad de Sevilla. 